\section{Christoph Bachmann}

\section{Einleitung}

Phytotherapie-Fachleute haben diesen Moment mit Ungeduld erwartet: Die Swissmedic hat grünes Licht für das Lavendelöl-Präparat Silexan WS 1265 (Kasten 1) gegeben; das Präparat ist registriert und ab sofort im Handel. Damit steht phytotherapeutisch tätigen Fachleuten eine pflanzliche Alternative zur Behandlung von Angst- und Spannungszuständen zur Verfügung. Die Registrierungsphase hat lange gedauert (siehe Interview in Kasten 2), doch nun steht das Arzneimittel zur Verfügung. Und in gewisser Weise kann man Silexan WS 1265 auch als Entschädigung oder ausgleichende Gerechtigkeit für das vor etwa 14 Jahren von den Registrierungsbehörden ausgesprochene und inzwischen wieder zurückgenommene Verbot von Kava-Präparaten betrachten - denn sowohl Kava-Präparate als auch Silexan WS 1265 wirken gegen Angst- und Spannungszustände. Das Kava-Verbot beruhte auf wenigen Fällen von Leberschädigungen, die möglicherweise mit der Einnahme von Kava im Zusammenhang standen. Diese wenigen Fälle stehen im Gegensatz zu den vielen dokumentierten Fällen, die im Zusammenhang mit der Einnahme von Benzodiazepinen standen.

\section{Indikationen}

Die in der Schweiz zugelassenen Indikationen sind «Ängstlichkeit» und

\title{
Lavendelöl-Präparat Silexan WS 1265 nun im Schweizer Handel - eine pflanzliche Alternative zur Behandlung von Angstzuständen
}

Mit der Registrierung des Lavendelöl-Präparates Silexan WS 1265 steht der Phytotherapie endlich wieder ein pflanzliches Arzneimittel zur Behandlung von Angststörungen zur Verfügung. Seit dem unrechtmässigen Verbot der Kava-Präparate Anfang dieses Jahrhunderts bestand bei dieser Indikation eine Lücke. Das identische Lavendelöl-Präparat ist seit 6 Jahren in Deutschland im Handel. In der Schweiz dauerte das Registrierungsverfahren hingegen fast 4 Jahre.

Kasten 1. Silexan WS 1265

Silexan WS 1265 ist eine definierte LavendelölZubereitung in Weichgelatine-Kapseln. Das Öl wird aus schmalblättrigem Lavandula angustifolia gewonnen. Silexan 1265 enthält weiter pharmazeutische Hilfsstoffe. 1 Kapsel enthält $80 \mathrm{mg}$ Lavendelöl. Die Hauptwirkstoffe des Öls sind Linalool und Linalylacetat. In der Schweiz ist Silexan 1265 unter dem Handelsnamen Lasea ${ }^{\circledR}$ im Handel.

«Unruhe». In Deutschland lautet die Indikation desselben Präparates «zur Behandlung von Unruhezuständen bei ängstlicher Verstimmung»; dies stellt also eine «härtere» Indikation als in der Schweiz dar.

Zwei renommierte PhytotherapieExperten haben in je einer klinischen Studie die Wirksamkeit von Silexan WS 1265 bei generalisierten Angststörungen dokumentiert. Eine generalisierte Angststörung ist eine generalisierte und anhaltende Angst, die nicht auf bestimmte Umweltbedingungen beschränkt ist, sondern das ganze Denken und Handeln der betroffenen Personen bestimmt. Diese sind oft nicht mehr in der Lage, ihren Alltag zu bewältigen. Hätte das Präparat in der Schweiz diese Indikation erhalten, wäre es in die Rezeptpflicht eingeteilt worden. Wenn ein Arzneimittel die Indikation GAD (Generalized Anxiety Disorder) erhält, ist es automatisch rezeptpflichtig. Und damit hätten die relativ vielen komplementärmedizinisch tätigen Apotheker und Apothekerinnen nicht die Möglichkeit gehabt, das Präparat einzusetzen.

\section{Studie 1: Kasper et al. [1]}

In dieser klinischen Studie dokumentiert Siegfried Kasper, Professor für Psychiatrie und Psychotherapie an der Universität Wien und ausgewiesener Experte für pflanzliche Arzneimittel zur Behandlung von psychischen Problemen, die Gleichwertigkeit von Silexan WS 1265 gegenüber Paroxetin zur Behandlung von generalisierten Angststörungen (GAD).

\section{Studiendesign}

Diese Studie wurde als randomisierte, doppelblinde, vierarmige Multizenterstudie angelegt sowie als Zweistufenstudie durchgeführt. Als erstes

\section{KARGER}

(C) 2017 S. Karger GmbH, Freiburg

Fax +497614520714 
Kasten 2. Interview mit Dr. Marco Würsch, Schwabe Pharma AG

Herr Dr. Würsch, es hat lange gedauert, bis das patentierte Lavendelöl Silexan WS 1265 in der Schweiz registriert werden konnte. Was waren die Hauptschwierigkeiten für die Registrierung?

Würsch: Die beiden grossen Knackpunkte waren die Indikation sowie die Abgabekategorie. Dies führte in beiden Fällen zu einem mehrmaligen Briefwechsel mit den Behörden. Im Mai 2016 wurde dann aber nach 3 Jahren und 8 Monaten die Zulassung für das neue Lavendelöl-Arzneimittel erteilt.

In Deutschland ist dieses Präparat schon einige Jahre im Handel. Hat diese Tatsache die Schweizer Registrierung nicht beschleunigt?

Leider nicht. Mit der Einführung des neuen Heilmittelgesetzes (HMG) ist dann aber eine vereinfachte Zulassung möglich und eine bestehende Zulassung in Deutschland wird stärker berücksichtigt.

Erleichtert eine EU-Zulassung eines Präparates bei der European Medicines Agency (EMA) in London die Registrierung in der Schweiz?

Wir haben für unser neues Produkt keine zentrale EU-Zulassung. Somit erübrigt sich diese Frage.

Entsprechen die in der Schweiz zugelassenen Indikationen «Ängstlichkeit» und «Unruhe» den Vorstellungen der Schwabe Pharma AG?

Wir mussten Kompromisse eingehen, um die Zulassung zu erreichen. Grundsätzlich sind wir aber mit der Indikation zufrieden, obwohl es Arzneimittel der Abgabekategorie D gibt, welche härtere Indikationen aufweisen. Gerne wären wir auch in der Kategorie D eingeteilt worden.

In der im Jahr 2014 publizierten klinischen Studie von Kasper et al. [1] erweist sich Silexan WS 1265 gegenüber Paroxetin als überlegen. In dieser Studie wurde die Hamilton Anxiety Rating Scale als Endpunkt bestimmt, nicht die Hamilton Rating Scale for Depression. Könnte man aber nicht trotzdem der Meinung sein, dass Silexan WS 1265 auch ein wirksames Antidepressivum sein könnte?

Eine Komorbidität zwischen Angststörungen und Depression ist in der Praxis mit ca. 30\% relativ häufig anzutreffen. Insofern könnte durchaus ein Zusammenhang bestehen. Wichtig ist aber zu wissen, dass Depression keine zugelassene Indikation des neuen Produktes aus Lavendelöl ist.

Ziel wurde die Überlegenheit von Silexan WS 1265 gegenüber Placebo überprüft, indem während der Studie die Verminderung des Wertes auf der Hamilton Anxiety Rating Scale (HAMA) gemessen wurde. In einem zweiten Schritt wurde die Wirksamkeit von Silexan WS 1265 mit derjenigen von Paroxetin verglichen.

Die für die Studie rekrutierten Probanden wurden in vier Gruppen mit zwei verschiedenen Dosierungen von Silexan, Paroxetin oder Placebo randomisiert und während 10 Wochen damit behandelt. Nach 2, 4, 6, 8 und 10 Wochen wurden die Wirksamkeit und die Sicherheit überprüft.

\section{Probanden}

Die für die Studie rekrutierten Probanden mussten zwischen 18 und 65 Jahre alt sein und eine Diagnose von GAD aufweisen (ICD-10; F41.1), deren HAMA-Gesamtwert $\geq 18$ betragen sowie weitere Bedingungen innerhalb der Erfassung des HAMAWertes erfüllen musste. Es wurden

Tab. 1. Behandlungsgruppen und Auswertungen

\begin{tabular}{lllll}
\hline & Behandlung & & & \\
\cline { 2 - 5 } & $\begin{array}{l}\text { Silexan 160 } \\
\mathrm{mg} / \text { Tag, } \mathrm{n}=128\end{array}$ & $\begin{array}{l}\text { Silexan 80 } \\
\mathrm{mg} / \text { Tag, } \mathrm{n}=135\end{array}$ & $\begin{array}{l}\text { Paroxetin 20 } \\
\mathrm{mg} / \text { Tag, } \mathrm{n}=137\end{array}$ & $\begin{array}{l}\text { Placebo, } \\
\mathrm{n}=136\end{array}$ \\
\hline Behandlungsverstösse & 23 & 16 & 29 & 18 \\
Behandlung korrekt durchgeführt & 105 & 119 & 108 & 118 \\
Sicherheitsauswertung & 121 & 135 & 137 & 136 \\
Keine Post-Baseline- & 7 & - & 5 & 1 \\
$\quad$ Wirksamkeitsdaten & 127 & 135 & 132 & 135 \\
Totalauswertung (Wirksamkeit) & 127 & & & \\
\hline
\end{tabular}

auch genaue Ausschlusskriterien definiert.

Von den ursprünglich 616 rekrutierten Probanden wurden 536 in eine der vier Behandlungsgruppen randomisiert. Ihre Baseline-Daten zeigten keine signifikanten Unterschiede.

Für die Sicherheitsauswertung wurden die Resultate aller Probanden verwendet, welche die Behandlung begonnen hatten. Für die Wirksamkeit wurden die Daten der Probanden ausgewertet, welche die Behandlung begonnen hatten und von denen PostBaseline-Daten über die Wirksamkeit vorlagen. Alle Dropouts wurden genau dokumentiert (Tab. 1).

\section{Endpunkte}

Primärer Endpunkt war die Abnahme des Totalwertes auf der HAMASkala von Baseline bis Behandlungsende, also nach Woche 10 oder bei Behandlungsabbruch im Falle einer vorzeitigen Beendigung der Behandlung.

Sekundärer Endpunkt waren die Anzahl der Responder und Remissionen definiert als ein verglichen mit Baseline mindestens 50\%iger Rückgang auf der HAMA-Skala sowie ein HAMA-Wert $<10$.

Weitere Endpunkte waren der Wert auf der HAMD (Hamilton Depression 
Tab. 2. Prozentuale Responderrate und Remission gemäss Studiendesign

\begin{tabular}{lll}
\hline & Responder, \% & Remission, \% \\
\hline Silexan WS 1265 160 mg/Tag & 60,3 & 46,3 \\
Silexan WS 1265 80 mg/Tag & 51,9 & 33,3 \\
Paroxetin 20 mg & 43,2 & 34,1 \\
Placebo & 37,8 & 29,6 \\
\hline
\end{tabular}

Scale), die Covi Anxiety Scale (CAS) und der klinische Gesamtzustand (Clinical Global Impression (CGI)).

\section{Resultate}

\section{HAMA}

Bei allen vier Behandlungsgruppen wurde zwischen Baseline und Studienende nach 10 Wochen eine Verminderung auf der HAMA-Skala ermittelt. Am meisten ausgeprägt war dies in den beiden Silexan WS 1265-Gruppen. Die Verbesserung der Gruppe mit $160 \mathrm{mg} /$ Tag war gegenüber Placebo ab der 4 . Woche und bei allen anderen Visiten signifikant $(p<0,01)$. Bei der Behandlungsgruppe mit $80 \mathrm{mg} /$ Tag war dies ab der 6 . Woche der Fall $(\mathrm{p}=0,02)$. Die Paroxetin-Gruppe erreichte gegenüber Placebo nach 6 Wochen fast Signifikanz $(p=0,06)$, nicht mehr aber nach 8 Wochen $(p=0,16)$ und am Studienende nach 10 Wochen $(\mathrm{p}=0,10)$.

In Bezug auf eine depressive Komorbidität zeigten die beiden SilexanGruppen eine klare und signifikante Wirksamkeit gegenüber Placebo, ebenso Paroxetin.

Eine Subauswertung verschiedener Aspekte von HAMA zeigte immer eine signifikante Überlegenheit der beiden Silexan WS 1265-Gruppen gegenüber Placebo. Paroxetin zeigte gegenüber Placebo eine signifikante Überlegenheit in Bezug auf die psychische Ängstlichkeit, nicht aber in Bezug auf somatische Ängstlichkeit. Bezüglich der Eigenbeurteilung der Patienten (CGI) zeigte sich bei allen drei aktiven Behandlungsgruppen gegenüber Placebo eine klare Verbesserung.

\section{Responderrate/Remission}

Die prozentuale Responderrate sowie Remission gemäss Studiendesign wird in Tabelle 2 aufgezeigt.

\section{Spezielle Auswertung}

Da die Anzahl der Probanden, welche die Studie vorzeitig beendeten, in der Paroxetin-Gruppe grösser war als in den beiden Silexan WS 1265-Gruppen, wurde eine Auswertung gemacht, in die nur die Probanden eingeschlossen wurden, welche die Studie vollständig beendet hatten und von denen somit keine Daten fehlten. Dies war der Fall in der:

- Placebo-Gruppe: 120/135 (88,9\%);

- Silexan 80 mg-Gruppe: 119/135 (88,1\%);

- Silexan 160 mg-Gruppe: 109/121 (90,1\%);

- Paroxetin-Gruppe: 108/132 (81,8\%).

Diese Auswertung bestätigte die Resultate der Silexan WS 1265-Gruppen in der Totalauswertung gänzlich. Für Paroxetin wurde durch diese Auswertung die Überlegenheit gegenüber Placebo in Bezug auf die Ängstlichkeit auf der HAMA-Skala sowie auf der CAS (beide $p<0,01$ ), bei verschiedenen Selbstbeurteilungen der Patienten hinsichtlich des Schweregrades der Erkrankung, auf den CGI usw. (p jeweils $<0,05$ ) bestätigt.

\section{Sicherheit}

Während der Behandlung berichteten folgende Probanden von unerwünschten Ereignissen (adverse events (AE)):

- 25,0\% in der Silexan-Gruppe
$160 \mathrm{mg} / \mathrm{Tag}$;
- 34,8\% in der Silexan-Gruppe $80 \mathrm{mg} /$ Tag;

- 40,9\% in der Paroxetin-Gruppe;

- 30,9\% in der Placebo-Gruppe.

Diese AE betrafen vor allem gastrointestinale Beschwerden, Infektionen sowie Beschwerden des Nervensystems. Von diesen insgesamt 281 gemeldeten AE erwiesen sich 5 als schwer. Der Zusammenhang mit der Behandlung wurde aber nicht bewiesen oder als unwahrscheinlich bezeichnet.

\section{Fazit}

Die vorliegende Studie zeigt die verglichen mit Placebo signifikante bessere Wirksamkeit von Silexan WS 1265 sowohl in der täglichen Dosierung von $160 \mathrm{mg}$ als auch von $80 \mathrm{mg}$ zur Behandlung von GAD. Beide Dosierungen verbesserten den allgemeinen mentalen Zustand der Probanden und zeigten auch einen Nutzen in Bezug auf die gesundheitsbezogene Lebensqualität. Weiter belegten sie eine klare Wirksamkeit hinsichtlich einer depressiven Komorbidität. Die Studie zeigt auch die Ebenbürtigkeit beider Silexan-Gruppen verglichen mit Paroxetin. Die in der Studie ermittelte Reduktion der HAMA-Werte nach 10 Behandlungswochen lässt sich mit der Reduktion von Bromazepam, Oxazepam, Escitalopram und Duloxetin vergleichen [2-4]. Beide Silexan-Dosierungen zeigten sich als sehr sicher.

Damit erweist sich das LavendelölPräparat Silexan WS 1265 als wirksame und sichere Behandlungsoption von GAD.

\section{Studie 2: Woelk und Schläfke [5]}

Diese Studie hat einen sehr ähnlichen Ansatz wie diejenige von Kasper et al. [1]. Sie vergleicht die Wirksamkeit von Silexan WS 1265 zur Behandlung von GAD mit Lorazepam, also einem Benzodiazepin, das unter anderem auch gegen GAD eingesetzt wird. 
Tab. 3. Durchschnittliche HAMA-Werte

\begin{tabular}{llllll}
\hline Behandlungsgruppen & \multicolumn{2}{l}{ Gesamt-Auswertung } & & \multicolumn{2}{l}{ Per Protocol-Auswertung } \\
\cline { 2 - 3 } \cline { 5 - 6 } & Baseline & nach 6 Wochen & & Baseline & nach 6 Wochen \\
\hline Silexan-Gruppe & $25 \pm 4$ & $13,7 \pm 6,7$ & & $25 \pm 4$ & $13,6 \pm 6,4$ \\
Lorazepam-Gruppe & $25 \pm 4$ & $13,4 \pm 6,6$ & & $25 \pm 4$ & $13,7 \pm 6,4$ \\
\hline
\end{tabular}

Ein kleiner Schönheitsfehler dieser Studie ist die ungewohnt niedrige Dosierung von Lorazepam, nämlich 0,5 mg. Die normale Dosierung von Lorazepam beträgt täglich $1-3 \mathrm{mg}$.

\section{Studiendesign}

Für diese 6 Wochen dauernde Behandlung wurden Personen zwischen 18 und 65 Jahren gesucht, bei denen eine GAD (gemäss DSM-IV 300.02) diagnostiziert wurde und welche einen HAMA-Wert $\geq 18$ sowie bei Punkt 1 «Ängstliche Stimmung» $\geq 2$ und bei Punkt 2 «Spannung» ebenso $\geq 2$ hatten. Rekrutierte Patienten, die während einer einwöchigen Auswaschphase mit Placebo behandelt wurden und trotzdem einen Rückgang auf der HAMA-Skala von mindestens $25 \%$ erlebten, wurden aus der Studie ausgeschlossen.

Dann folgte eine 6-wöchige Behandlungsphase, während der die Probanden entweder täglich $80 \mathrm{mg} \mathrm{Si-}$ lexan WS 1265 oder $0,5 \mathrm{mg}$ Lorazepam erhielten. Weiter erhielt die Silexan-Gruppe ein Lorazepam-Placebo und die Lorazepam-Gruppe ein Silexan-Placebo.

\section{Zielvariablen}

Primäre Zielvariable war die Veränderung auf der HAMA-Skala von Baseline bis zum Studienende nach 6 Wochen.

Sekundäre Zielvariablen waren die Anzahl der Responder, definiert als eine mindestens 50\%ige Reduktion des HAMA-Wertes. Als Remission wurde eine Reduktion unter einen HAMA-Wert von 10 definiert.
Als weitere Parameter wurden der CGI, die Zung Self-Rating Anxiety Scale (SAS), der Penn State Worry Questionnaire (Version vergangene Woche (PSWQ-PW)), der «SF-36 Health Survey»-Fragebogen für den Mentalzustand sowie ein Schlaftagebuch der Probanden beigezogen.

Für die Studie wurden 78 Personen rekrutiert, von denen 77 randomisiert wurden, und zwar 40 in die Silexan WS 1265- und 37 in die LorazepamGruppe. 38 Probanden der SilexanGruppe sowie 36 Probanden der Lorazepam-Gruppe beendeten die Studie. Für die Gesamt-Wirksamkeitsauswertung wurden alle in die Behandlung aufgenommenen Probanden und für die Per-Protocol-Auswertung 36 Patienten der Silexan-Gruppe sowie 33 Patienten der Lorazepam-Gruppe verwendet. Alle Dropouts wurden genau dokumentiert.

\section{Resultate}

Primäre Zielvariable: Veränderung auf der HAMA-Skala

Hinsichtlich der primären Zielvariable wurde in beiden Behandlungsgruppen eine deutliche Verminderung der Werte auf der HAMA-Skala ermittelt: Die Werte unterschieden sich in Bezug auf die Gesamt-Auswertung und die Per-Protocol-Auswertung kaum (Tab. 3).

Diese Zahlen dokumentieren die Nichtunterlegenheit von Silexan gegenüber Lorazepam bezüglich der Wirksamkeit zur Behandlung von GAD.

\section{Probanden}

Sekundäre Zielvariable: Responder/ Remission

21 Probanden $(52,5 \%)$ in der Silexan-Gruppe sowie 15 (40\%) in der Lorazepam-Gruppe erfüllten die Bedingungen als Responder, d.h. eine Verminderung des HAMA-Wertes um mindestens 50\%. Die Nichtunterlegenheit von Silexan WS 1265 gegenüber Lorazepam war damit signifikant $(p=0,04)$. Am Ende der aktiven Behandlung konnte bei 16 Probanden (40\%) der Silexan-Gruppe sowie bei 10 Probanden (27\%) der LorazepamGruppe eine Remission festgestellt werden. Auch hier erwies sich Silexan WS 1265 gegenüber Lorazepam als signifikant nicht unterlegen $(\mathrm{p}=0,04)$.

Sehr ähnliche Zahlen ergab die Auswertung der Verbesserung in Bezug auf somatische und psychische Ängstlichkeit. Die Baseline-Werte der beiden Behandlungsgruppen waren vergleichbar, ebenso die Verbesserung nach 6 Wochen aktiver Behandlung.

\section{Sekundäre Zielvariable: PSWQ- \\ $P W$, SAS und SF-36-Mentalzu- stand}

Auch alle anderen Resultate der sekundären Zielvariablen, d.h. PSWQPW, SAS und SF-36-Mentalzustand, zeigten ähnliche und deutliche Verbesserungen für beide Behandlungsgruppen.

\section{Sekundäre Zielvariable: CGI}

Bei Baseline wurden in beiden Behandlungsgruppen die meisten Patienten als moderat bis stark krank eingestuft. Nach 6 Behandlungswochen wurden 24 Patienten (60\%) der Silexan-Gruppe nur noch als sehr mild krank eingestuft. In der LorazepamGruppe waren dies 19 Patienten (51,3\%).

\section{Sicherheit}

Während der Studie wurden keine schwerwiegenden AE dokumentiert. 
In der Silexan-Gruppe berichteten 20 Probanden von 26 AE; in der Lorazepam-Gruppe wurden bei 18 Probanden 19 AE dokumentiert. Bei 11 der AE in der Silexan-Gruppe konnte kein kausaler Zusammenhang mit der Behandlung festgestellt werden, jedoch bei $7 \mathrm{AE}$ in der Lorazepam-Gruppe. In der Silexan-Gruppe betraf die Mehrheit der AE den Gastrointestinaltrakt. In der Lorazepam-Gruppe wurden ein Fall von Schwindel und 6 Fälle von Müdigkeit gemeldet, was typische AE für Lorazepam sind.

\section{Fazit}

Auch diese Studie dokumentiert die Wirksamkeit und Sicherheit des Lavendelöl-Präparates Silexan WS 1265 und zusätzlich die Nichtunterlegenheit von Silexan WS 1265 gegenüber (niedrig dosiertem) Lorazepam. Weiter weist Silexan WS 1265 weniger AE auf als Lorazepam.

\section{Literatur}

1 Kasper S, et al: Lavender oil preparation Silexan is effective in generalized anxiety disorder - a randomized, double-blind comparison to placebo and paroxetine. Int J Neuropsychopharmacol 2014;17:859-869.

2 Woelk H, et al: A comparison of Kava special extract WS 1490 and benzodiazepine in patients with anxiety. Healthnotes Rev 1999;6: 265-270.
Damit erweist sich das LavendelölPräparat als plausible Alternative $\mathrm{zu}$ synthetischen Arzneimitteln bei der Behandlung von GAD.

\section{Bedeutung für die Schweiz}

Mit der Registrierung des Lavendelöl-Präparates Silexan WS 1265 ist in der Schweiz eine komplementärmedizinische Lücke geschlossen worden. Anfang dieses Jahrhunderts wurden die Kava-Präparate verboten - zu Unrecht, wie kürzlich ein deutsches Gericht festhielt. Alkoholische Kava-Extrakte dürfen wieder in den Handel gebracht werden. Doch ob diese je wieder ihre ursprüngliche Bedeutung zurückgewinnen werden, steht auf einem anderen Blatt geschrieben.

Nun steht jedoch PhytotherapieFachleuten ein Lavendelöl-Präparat zur Verfügung. Nach einem langen Registrierungsverfahren, das natürlich viel Geld kostete, hat die Swissme- dic, die schweizerische Registrierungsbehörde, grünes Licht für die Marktzulassung der Lavendelöl-Zubereitung Silexan WS 1265 gegeben. Als Indikation wurden Ängstlichkeit und Unruhe zugelassen. Das ist natürlich weit weg von der Wirksamkeit «GAD», die in den beiden in diesem Artikel zusammengefassten Studien dokumentiert werden konnte. Doch eine solche Indikation würde das Präparat natürlich unter Rezeptpflicht stellen, sodass es nur von Ärzten eingesetzt werden könnte, die sich mit Phytotherapie befassen, nicht aber von Apothekern und Apothekerinnen. Mit den beiden vorliegenden Indikationen ist die Firma Schwabe dennoch recht zufrieden, weil damit Silexan WS 1265 rezeptfrei ist und damit auch in Apotheken empfohlen werden darf.
3 Bielski RJ, et al: A double-blind comparison of escitalopram and paroxetine in the long-term treatment of generalized anxiety disorder. Ann Clin Psychiatry 2005;17:65-69.

4 Allgulander C, et al: Pharmacotherapy of generalized anxiety disorder: results of duloxetine treatment from a pooled analysis of three clinical trials. Curr Med Res Opin 2007;23:12451252.
5 Woelk H, Schläfke S: A multi-center, doubleblind, ramdomised study of the Lavender oil preparation Silexan in comparison to Lorazepam for generalized anxiety disorder. Phytomedicine 2010;17:94-99. 